\title{
Analysis of Public Health Emergency Mechanism
}

\author{
Min Cheng, Mingjun Han
}

Aba Teachers University, Sichuan Wenchuan 623002

Keywords: Public Health, Emergency Issue, Improvement Mechanism

\begin{abstract}
Public health emergencies are major infectious disease epidemics, mass unexplained diseases, major food poisoning and occupational poisoning, and other events that seriously affect public health, which occur suddenly or may cause public health damage. It is difficult to simply make a classic summary of the health emergency machine. In academic and practical work, the expression of the health emergency mechanism is mostly health emergency management mechanism, health emergency operation mechanism, or health emergency response mechanism. These definitions and generalizations only highlight a certain aspect of the health emergency mechanism or a certain cross section in the system from the perspective of function and effect. They fail to outline the connotation of a clear health emergency mechanism as a whole, and simply take one of the essentials to represent health. The emergency mechanism will limit our thinking. It is difficult to break through the existing rules and regulations in academic discussions and practical work. It is difficult to obtain important theoretical innovations and it is even more difficult to guide China's health emergency work.
\end{abstract}

\section{Introduction}

Public health emergencies refer to major infectious disease epidemics, mass unexplained diseases, major food and occupational poisoning, and other events that seriously affect public health, which occur suddenly, cause or may cause serious damage to public health. Its characteristics include suddenness, publicity, harmfulness, internationality, and comprehensive and systematic treatment. In recent years, public health emergencies affecting human health and even life safety have occurred from time to time. They not only threaten people's lives, damage physical and mental health, but also have a serious impact on the social economy. Therefore, how to deal with the public health emergency, the role of the emergency mechanism is very important. However, in recent years, many problems have been exposed in the process of building emergency response mechanisms for public health emergencies.

\section{Problems in the construction of emergency mechanism for public health emergencies in China}

At present, event monitoring is mainly based on passive monitoring, lacking corresponding incentive mechanisms and hard constraints, resulting in a large time lag for event reporting and analysis; poor information channels, lack of unified standards and sharing mechanisms, and inability to make existing data, Information is an emergency decision-making service; China has not yet established a scientific and effective forecasting and early warning method based on the information reporting network system, which cannot meet the needs of forecasting and early warning and emergency response of public emergencies.

At present, event monitoring is mainly based on passive monitoring, lacking corresponding incentive mechanisms and hard constraints, resulting in a large time lag for event reporting and analysis; poor information channels, lack of unified standards and sharing mechanisms, and inability to make existing data, Information is an emergency decision-making service; China has not yet established a scientific and effective forecasting and early warning method based on the information reporting network system, which cannot meet the needs of forecasting and early warning and emergency response of public emergencies. 
Despite the continuous deepening of reforms by medical and disease control agencies, the requirements for effective handling of various major public health emergencies in terms of mechanisms, especially capacity and team building, are far apart. In the municipal and county-level institutions, there is a lack of emergency protection and emergency training. In many places, some disease control agencies are also facing basic survival problems. The protective equipment and special effects of rescue workers are lacking, and the brain drain is serious.

The scientific and stable investment mechanism for emergency work has not yet been established. There is widespread re-construction, light maintenance and operation, and emergency functions cannot be fully utilized. In response to public health emergencies, there is no normal channel for emergency compensation for financial compensation. The funds are lagging behind. At the same time, there is a danger of misappropriation, seizure and evaporation. There is a lack of supervision and evaluation mechanism for financial compensation funds.

There is no unified system for the construction of emergency plans for public health emergencies at the provincial, city and county levels. There is a lack of good connection and coordination between the plans. The content overlaps and vacuums coexist, and there are many loopholes in the technical links. Some of the plans are outdated and urgently needed to be revised. We will improve the laws and regulations related to the preparation of emergency plans, such as the disaster relief and disease prevention plan, and the laws and regulations related to the preparation of emergency plans. The publicity and education of emergency plans are not enough. In the grassroots emergency work, the plans often become furnishings or processed according to past experience. Public health emergencies. Over the years, China has gradually formed a crisis management model based on departmental division. The relationship between the departments and departments is asymmetric. The "interface relationship" between various crisis management departments is vague, and they are independent and lack coordination. Information exchange between functional departments and regions is not smooth, and scattered and scattered information is difficult to integrate, inherit, analyze, and deal with. Health emergency research lags behind actual needs, the size and direction of scientific research funds are lost, scientific research methods are backward, and standardization is lacking. Due to the division of administrative system, health emergency research work is disorderly, scientific research management system is not smooth, resulting in overlapping institutions. Dispersion of power, unclear division of management power, poor information and difficulty in resource integration, and uncoordinated actions of the entire scientific research work. The laws and regulations between departments are not connected, the standards are not uniform, the responsibilities are unclear, the operation links are complicated, the administrative costs are high, the efficiency is low, and the problems of rapid response are still outstanding. Although the emergency response law has been introduced However, there are still many legal issues in the operational level of actual work.

\section{Establish and improve mechanisms and measures for responding to public health emergencies}

The early warning and handling of public health emergencies are related to the vital interests of the broad masses of the people. At this stage, although the state has increased relevant investment, the total amount is far from sufficient, far below the economic development, and cannot meet the needs of the broad masses of the people. Therefore, the state and the government should increase health investment according to the actual situation of the region. In particular, it should increase investment in grassroots emergency agencies, strengthen the construction and equipment of facilities and equipment, train emergency personnel, and improve relevant emergency systems.

Establish an emergency response system that combines with local realities to better prevent and handle emergencies and ensure social stability, and governments at all levels should establish a unified command system. Comrade Wen Jiabao stressed at the symposium on the implementation of the "Emergency Regulations on Public Health Emergencies" that the handling of public health emergencies should be based on the principle of unifying leadership, grading and responsibility, timely response, decisive measures, relying on science, and strengthening cooperation. To establish 
a rapid response network system for responding to emergencies, government departments should give full play to their functions and manage them according to the principle of localization. At the same time, the various components involved in the construction should clarify their tasks and responsibilities for prevention. And establish a corresponding coordination mechanism, highlight the principle of standardized management according to law, and establish a management system with legal and regulatory support system.

By using computer Internet, video image transmission technology, wired or wireless communication, and GIS, the existing disease control information systems at the national, provincial, municipal, and county levels will be linked to initially establish a public emergency that can cover the whole country. The health emergency command platform basically realizes resource integration and resource sharing of public health emergency information that can be controlled by the domestic disease control system, and connects with the health supervision department and the health permit department to establish and improve the emergency public health emergency information platform. To achieve a rapid response to public health emergencies. In addition, it is necessary to establish a well-functioning health information monitoring system. In addition to routine routine monitoring, scientific analysis and comprehensive evaluation of monitoring data results, it is more important to be able to dynamically evolve trends and society for some major infectious diseases. The overall state of change in public health is monitored. Actively monitor and promptly alert public health emergencies, identify the signs of emergencies at the first time and analyze and judge their development trends, identify hidden dangers as early as possible and determine the nature and extent of public health emergencies so that they can suddenly When public health incidents come, they can propose reasonable solutions and quickly formulate and take countermeasures to avoid or reduce the losses and harms caused by public health emergencies.

In order to effectively prevent and control the occurrence of public health emergencies and maintain social stability and economic development, an effective response plan must be formulated. The formulation of the plan should be more specific and more operative. It can ensure timely and effective response after the occurrence of public health emergencies, and can properly handle and control, and ultimately the economic losses of public health emergencies. Personnel casualties are kept to a minimum. At the same time, the plan should also be implemented and managed in an organized manner, such as the principles of prevention and treatment, medical treatment, epidemic monitoring and control, laboratory testing, related policies and information release management, etc., and should also support personnel, technology and material security. .

Optimize the construction of talent echelon, promote and accelerate the reform of the personnel system, create a good living environment and conditions for the outstanding emergency management and disposal of talents; at the same time, strengthen the business training of relevant practitioners, encourage employees to receive education and re-education, Enrich and accumulate coping skills in an emergency. In addition, if conditions permit, you can introduce or hire professional and technical personnel in the industry, and grasp the development trends in the field of emergencies in the international and domestic areas in a timely manner, so as to establish a rush to come and come, The emergency response team that can win the battle is an important component of the response to public health emergencies.

The construction of a public health environment requires the participation of the whole society and the active publicity of the media. At the same time, it must establish an information disclosure system for public health emergencies. In this regard, when the media cooperates with the government to publish relevant information, it should strive to be timely, proactive, realistic, accurate, and effective. It is strictly forbidden to speculate on the basis of partial and unreasonable speculation, so that the public can keep abreast of the development of emergencies and The local people are effectively channeled to avoid unnecessary panic. Only through the positive guidance of the media and the active publication of government website information can the public understand the progress of emergencies in the first time, in order to better respond to and cope with the emergency measures taken by relevant departments, so as to prevent them from being strictly guarded. Control the development of events. 


\section{Conclusion}

In the 21st century, public health emergencies in China have occurred frequently, and melamine, SARS, hand, foot and mouth disease are hard to prevent. Therefore, only by actively establishing and improving the emergency response mechanism for public health emergencies, when the disaster suddenly drops, we can make it happen and change.

\section{Acknowledgements}

1) Sichuan Provincial Department of Education Humanities and Social Sciences Key Research Base - Sichuan Leisure Sports Industry Development Research Center Funded Project (XXTYCY2017C07): Sichuan Province "Internet + Ethnic Area Leisure Sports" Industry Research (Aba Prefecture as an example);

2) Aba Normal College Key Program (ASA18-12) Under the background of "healthy pension", the training of sports health professional training and Sanjiang, Shuimo, Xuankou elderly health tourism tourism school;

3) Aba Teachers College (ASB18-17): Research on AIDS prevention and control strategies in colleges and universities in Sichuan (taking Aba Teachers College as an example).

\section{References}

[1] Ma Feicheng, Zhang Qin. Hotspots of Knowledge Management Research at Home and Abroad_-Statistical Analysis Based on Word Frequency[J].Journal of the China Society of Scientific Information,2006,25(4):163.

[2] Hu Wenkui. Reflections on hospital response to public health emergencies [J].Guangdong Medical Journal, 2003, 24(6):145.

[3] Chen Hongyu. "Reinforcement of the Dead" or "Preparing for the Rain" - The establishment of a public health response system from the sudden public health incident [J]. Pharmaceutical World, 2004, (3): 8.

[4] Chu Mingwei, Ding Xiaodan. The establishment of the mechanism of the Chinese government's response to public health emergencies [J]. Journal of Xinxiang Medical College, 2004; 21(6): 511.

[5] Health Emergency Office of the Ministry of Health. 2007 Health Emergency Work Points [J]. China Public Health Management, 2007; 23(1): 4. 